\title{
A NOTE ON FROBENIUS NORM PRESERVERS OF JORDAN PRODUCT
}

\author{
Bojan KuZma and Tatjana Petek
}

Abstract. We classify maps on $n$-by- $n$ complex matrices which preserve the Frobenius norm of Jordan product.

Mathematics subject classification (2010): 15A60, 15A86, 15A30.

Keywords and phrases: Jordan product, Frobenius norm, isometry.

\section{REFERENCES}

[1] A. Fošner, B. Kuzma, T. KuZma, N.-S. Sze, Maps preserving matrix pairs with zero Jordan product, Linear Multilinear Algebra 59, 5 (2011), 507-529.

[2] R. Horn, C. Johnson, Topics in Matrix Analysis. Cambridge UP, 1991.

[3] B. Kuzma, G. LeŠnjak, C.-K. Li, T. PeteK, L. Rodman, Conditions for linear independence of two operators. Operator Theory: Advances and Applications 202 (2010), 411-434.

[4] B. Kuzma, G. LešnJak, C.-K. Li, T. PeteK, L. Rodman, Norm preservers of Jordan products, Electron. J. Linear Algebra 22 (2011), 959-978. 